\title{
Drawing to Extend Waddington's Epigenetic Landscape
}

Gemma Anderson, Berta Verd, and Johannes Jaeger*

Gemma Anderson: University of Exeter, g.e.anderson@exeter.ac.uk, ORCID ID: 0000-00026678-5109. www.gemma-anderson.co.uk

Berta Verd: University of Cambridge, berta.verd@ gmail.com

Johannes Jaeger: Konrad Lorenz Institute for Evolution and Cognition Research (KLI), Klosterneuburg, Austria yoginho@gmail.com, ORCID ID: 0000-0002-2568-2103.

* Current affiliation: Complexity Science Hub (CSH) Vienna, Austria

(C) ISAST

Manuscript received 10 November 2017.

\begin{abstract}
We describe a collaboration between an artist, a mathematician, and a biologist, which examines the potential of drawing for understanding biological process. As a case study, it considers C. H. Waddington's powerful visual representation of the "epigenetic landscape," whose purpose it is to unify research in genetics, embryology, and evolutionary biology. We explore the strengths, but also the limitations of Waddington's landscape and attempt to transcend the latter through a collaborative series of exploratory images. Through careful description of this drawing process, we touch on the epistemological consequences it had on all participants, artist and scientist alike.
\end{abstract}

\section{Introduction}

When artists have tried to learn direct lessons from science, copying the visual phenomena turned up by scientific research or technically based industry, not much of value or profundity has been produced. The notions which have been more fructifying are those which have been absorbed by empathy, through the pores, as it were. And they have been expressed again by the artists not so much in any explicit exposition or diagramming of scientific ideas, but rather by living a life of implicit incorporation into a work of art - an artifact - from which the spectator again absorbs them by in-feeling more than by analysis. It is at the deep levels of the human psyche, where these kinds of communications operate, that there is the closest unity between science and art. [1]

This paper presents a transdisciplinary collaboration between a visual artist (Anderson), a mathematician (Verd), and a biologist (Jaeger). It aims to realize and highlight the potential of drawing for understanding biological process. Specifically, we use drawing as an epistemic tool to generate new images to think with [2].

In the very first volume of Leonardo, published in 1968, Waddington tells us that "science is something more than a collection of conceptual or practical results. It is also an activity; and its practice involves, as a very important part, the exercise of the faculties of insightful perception of natural phenomena and of the imaginative creation of new concepts" [3]. In fact, both scientists and artists have to be able to handle concepts before they are fully and explicitly formulated, a 
kind of pre-linguistic exploration of ideas. The release from scientific constraints in artistic practice makes collaborative image-making a mind-opening experience that can be mutually beneficial for scientist and artist alike.

Here, we build on previous epistemological inquiries of Anderson [4] as we develop and demonstrate the use of drawing in representing biological process. Our approach is informed by Anderson's experience of morphological and topological drawing, combined with Verd's training in dynamical systems theory and evolutionary developmental biology, and Jaeger's process-based approach to the study of living systems. We integrate methods by artists such as Paul Klee, who have pioneered techniques for the representation of dynamics [5]. Alongside the practical development of drawing methods to this end, we aim to generate and stimulate discussion about the epistemological value of drawing, thus sustaining and re-vitalising the practice of hand-drawing in contemporary scientific practice.

Our different but complementary perspectives brought us to reconsider and extend one of the most powerful visual representations of biological process: Conrad Hal Waddington's "epigenetic landscape" (Fig. 1) [6]. Beyond visualizing the paths taken by organisms during their embryonic development, this iconic image has the ambition to reunify embryology with genetics and evolution by showing how complex genetic changes affect the topography of the landscape. Here, we tell the story of creating an extended version of this representation [7] through a series of six collaborative images, which identify and address challenges for visualizing the complex, multi-dimensional dynamics of evolving biological systems.

\section{Waddington's Landscape: Influence and Limitations}

Waddington's visualization works as follows: there is a ball on an abstract surface. This ball represents the current state of a developing biological system. It rolls downhill along valleyscalled chreodes - that determine the possible trajectories the system can take. There are branching points in these valleys, one for each developmental decision, for example, whether to become a neuron or a skin cell. Each valley is bounded by steep slopes, keeping the ball on track as it is subjected to perturbations that push it away from its default path. This represents the robust or, as Waddington calls it, canalised nature of development [8]. The landscape is not fixed: If we peek underneath the surface, we see pegs that connect to it by a network of guy ropes, which pull and alter the topography in complex, non-intuitive ways. The pegs represent genes, and the webbed ropes their influence on the developmental system. In this way, Waddington graphically combines developmental dynamics, the ball rolling down the landscape, and evolutionary dynamics, the landscape shifting underneath the ball.

While the extent of Waddington's conceptual impact on developmental and evolutionary biology is debated, his pictorial legacy is wide-ranging and profound [9]. His landscape is used to integrate and structure thinking across disciplinary boundaries. René Thom put Waddington's visual intuitions on a firm mathematical basis [10]. More recently, landscape images have become widespread in stem cell biology, where they connect experimental molecular biology with systems-level models [11]. In general, Waddington's landscape is used by researchers that apply dynamical systems theory to the study of developmental or other regulatory processes and their evolution [12]. In all these cases, the landscape brings into visual focus the particular 


\section{(c) 2019 ISAST}

dynamic nature of biological processes, and facilitates the transdisciplinary communication required to study these dynamics in an integrative and systematic way.
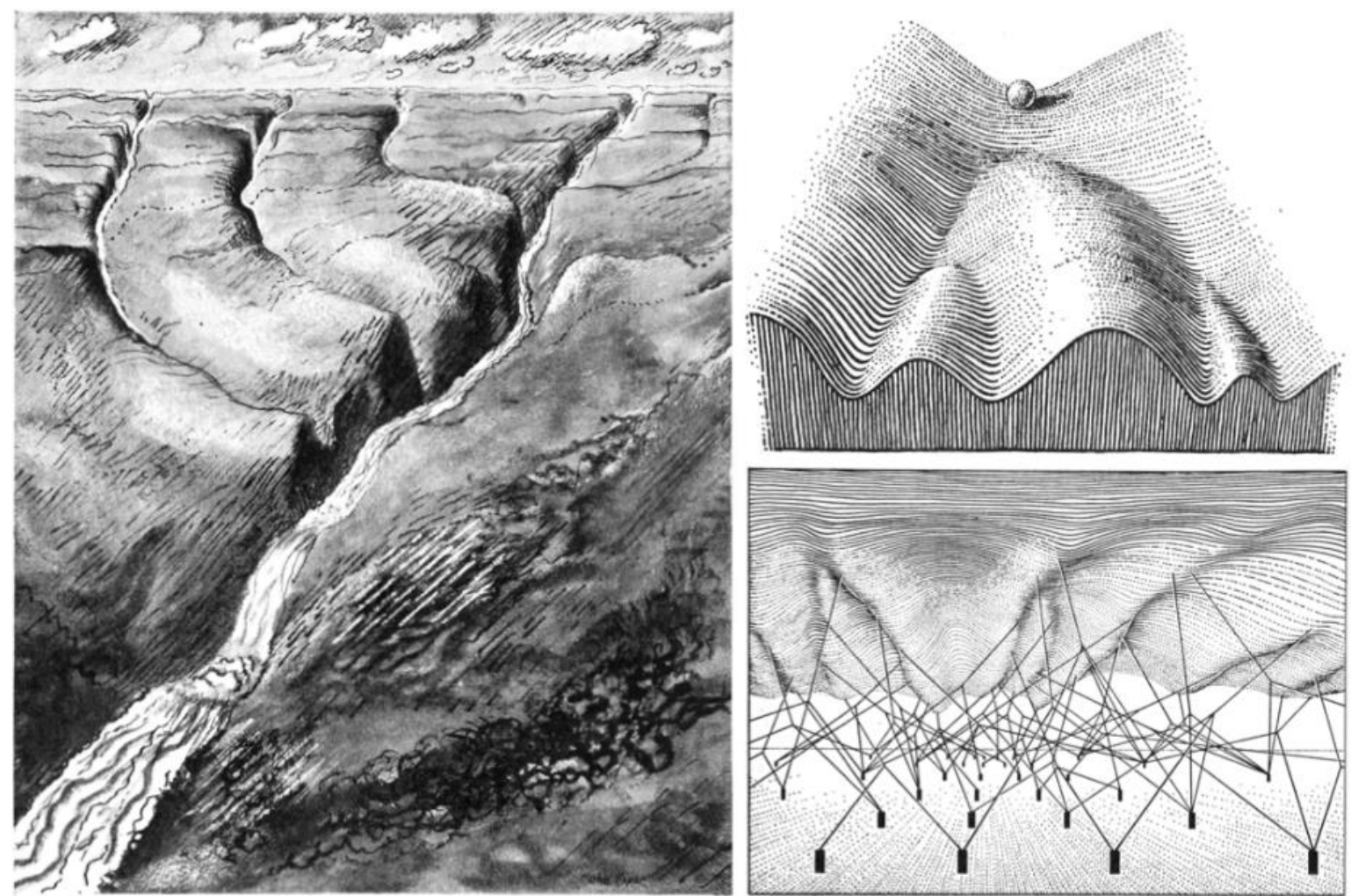

Fig. 1. Waddington's epigenetic landscape. Left: drawing by John Piper for Waddington's 'Organisers \& Genes" (1940, Cambridge: Cambridge University Press). The original caption reads: "Looking down the valley towards the sea. As the river flows away into the mountains it passes a hanging valley, and then two branch valleys, on its left bank. In the distances the sides of the valleys are steeper and more canyon-like." Right: Waddington's classical depictions of his landscape from "Strategy of the Genes" (1957, London: Allen \& Unwin). Top right: top view of the landscape where the path followed by the ball represents a developmental trajectory (or chreode). The valleys represent different alternative paths to depict different developmental potentials, for example, differentiation leading to various cell types. Bottom right: by depicting the underside of the surface, Waddington illustrated the idea that genes can change the landscape during evolution. The pegs at the bottom represent genes acting on the landscape by tugging on the intertwined web of guy ropes thereby changing the topography of the valleys and ridges above. (Images courtesy tandfonline.com)

These examples highlight that Waddington's landscape was successful in a role that would have pleased its originator: it stimulates visual thought, enabling us to contemplate biological processes in new ways which are not yet explicitly conceptualized. To paraphrase Waddington: his artful representation helps to "loosen the joints of the scientist's imagination", creating a space for the associative play required to introduce new concepts in theory formation [13].

Despite its success, Waddington's landscape suffers from several important limitations. First of all, it may be too abstract to be helpful, visually informative, or imaginatively stimulating, and it is certainly difficult to connect to the experimental study of specific morphogenetic processes [14]. For example, it is not clear how the position of the ball within the landscape-representing the state of the developing system - connects to morphological changes or other indicators of 


\section{(c) 2019 ISAST}

system state that can be observed and measured in the laboratory. Moreover, the particular topography Waddington uses in his illustrations is intuitive, but does not hold up under mathematical scrutiny [15].

Finally, and most importantly in our context, Waddington's landscape cannot visualize a key property of biological systems: his two-dimensional representation of the topographical surface does not accommodate complex behaviour, such as oscillatory dynamics. In the landscape, the ball always has to run downhill, while in oscillatory dynamics, the system revisits the same state or topographical location periodically. Oscillations are at the core of many fundamental cellular and developmental processes [16]. One important example of oscillatory patterning is somitogenesis, the process by which vertebrate animals form their body segments (called somites) — adding them one by one as they grow longer during embryogenesis [17]. We use this developmental process as biological anchor and motivation for our own creative process.

\section{Extending Waddington's Landscape through Collaborative Image-Making}

We explore the limits of Waddington's landscape, and extend it to accommodate oscillatory and other complex dynamics, through a process of collaborative image-making that involves artist and scientist in equally crucial roles. Here, we reflect on the dynamics of this collaboration. We connect back to the epistemological nature of drawing, emphasising its role in transdisciplinary communication and the formulation of new concepts. Finally, we discuss how our collaborative process informs both artistic and scientific methodology for the study and understanding of biological process.

We develop our collaboration through a series of six images - each a unique process of creating and exchanging knowledge through drawing. Iterative in nature, each image builds on critical discussions of the previous one.

The process began in September 2016, when Anderson was visiting the Konrad Lorenz Institute for Evolution and Cognition Research (KLI) in Klosterneuburg near Vienna. She had been invited by Jaeger, whose interest was piqued by meeting with Anderson at a process philosophy workshop and discovering her depiction of morphological transformation in her 'Isomorphogenesis' project. Isomorphogenesis is an exercise in theoretical morphology consisting of a series of drawings that represent an analogue simulation of the dynamic possibilities of form (Fig. 2). It integrates D'Arcy Thompson's grid transformations, Klee's colour gradation method, and William Latham's "FormSynth" system for the generation of form [18]. What is missing from the Isomorphogenesis series is a Waddington-style landscape shaping and constraining the morphological transitions taking place. This observation provides a natural starting point for our exploration. 


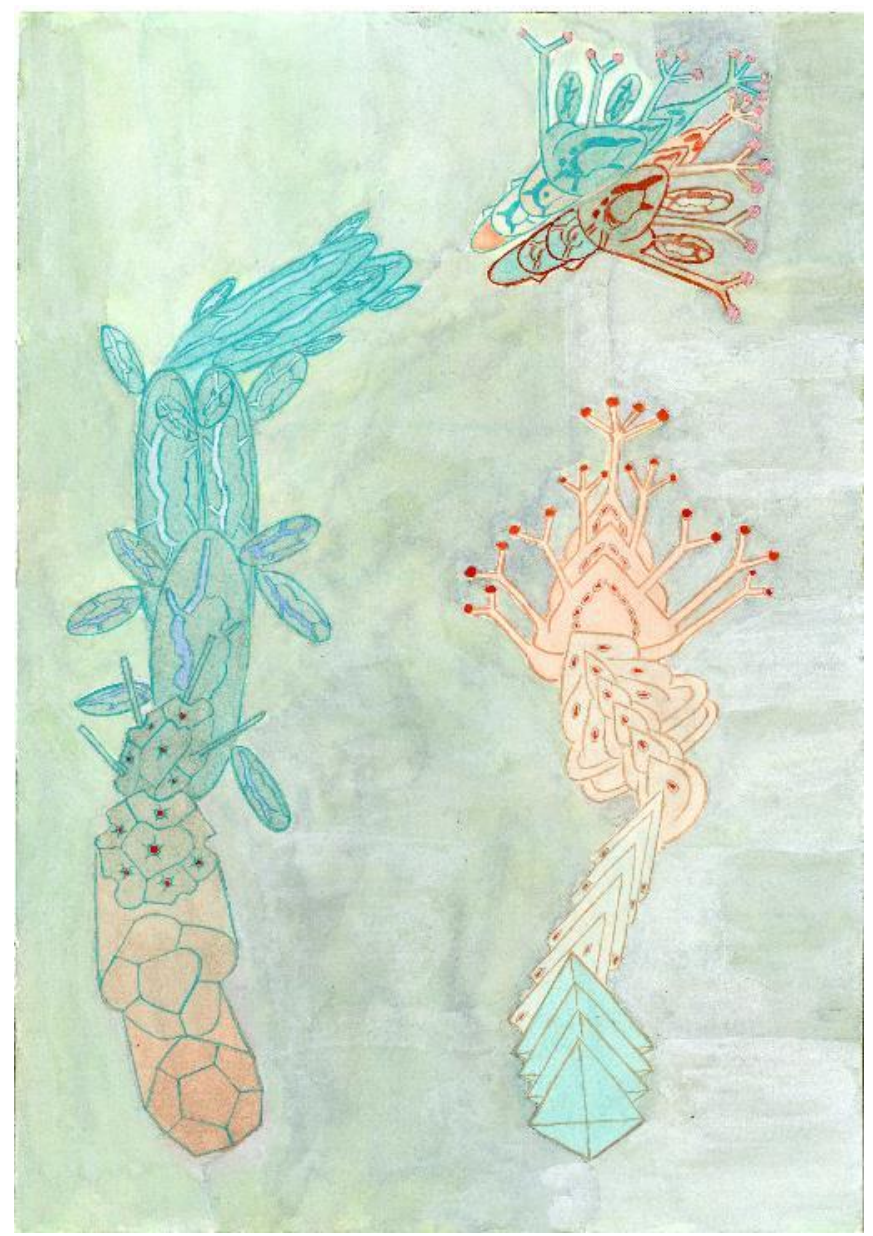

Fig. 2. Gemma Anderson: "Isomorphogenesis No.2.", watercolour on paper, 2014. Image from the Isomorphogenesis series, which explores the potentialities of representing morphology as a dynamic and formative process. (C) Gemma Anderson)

The ball in Waddington's landscape (Fig. 1) remains the same over time as it rolls down its valley. It does not undergo any morphological transformations. We imagined overcoming this limitation by replacing the ball moving through the landscape with an Isomorphogenesis transformation. The resulting experimental image by Anderson is shown in Fig. 3. It reduces the level of abstraction of Waddington's representation by explicitly showing the trajectory of morphological change (the chreode) through the valleys of the landscape. Vice versa, it provides context for the developmental processes in the Isomorphogenesis series through its explicit depiction of the landscape. However, it remains unsatisfactory, since it is still limited to a twodimensional landscape surface, which restricts its ability to portray complex developmental processes more realistically. 


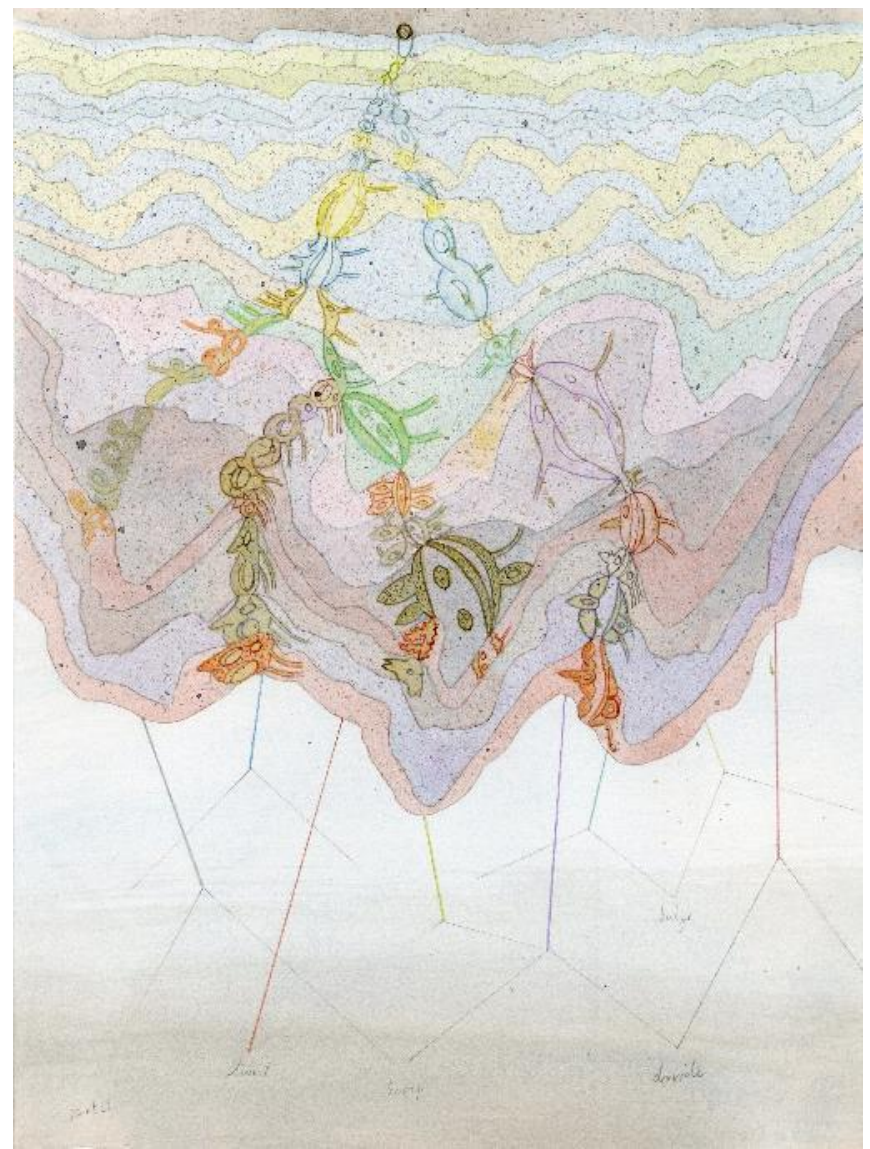

Fig. 3. Gemma Anderson, "Isomorphogenesis embedded in Waddington's Epigenetic Landscape." Watercolour on paper, 2016. (C) Gemma Anderson)

Taking inspiration from Anderson's and Verd's mutual interest in topology, they decided to experiment with different geometrical structures as landscapes on which to draw developmental processes. The idea is that topologically complex landscapes would allow us to depict an expanded range of developmental dynamics. In particular, we wanted to find a landscape topology enabling us to represent the oscillatory dynamics involved in somitogenesis (Fig. 4). The addition of somites to the growing vertebrate body involves repeating waves of genes being activated and repressed, creating oscillatory patterns of gene product synthesis, and hence dynamic travelling waves of cell state changes moving through the tissue [19]. How to represent such periodic oscillations? A torus is the natural surface topology for this purpose (Fig. 5).

In this image, called 'Somitogenesis/Oscillations:Torus', the developmental trajectory wraps around the torus like a string. The chreode is now free to oscillate. Its cyclic colour gradation represents the oscillating levels of gene products involved in somitogenesis. A new somite is formed after each one of these cycles, elongating the embryonic axis one segment at a time. We have mentioned above that chreodes are canalised, that is, buffered against perturbations, which Waddington summarizes under the concept of "developmental noise." Anderson integrates a visual interpretation of such noise using artistic methods such as watercolour speckling, splashes, and gestures in the image. 

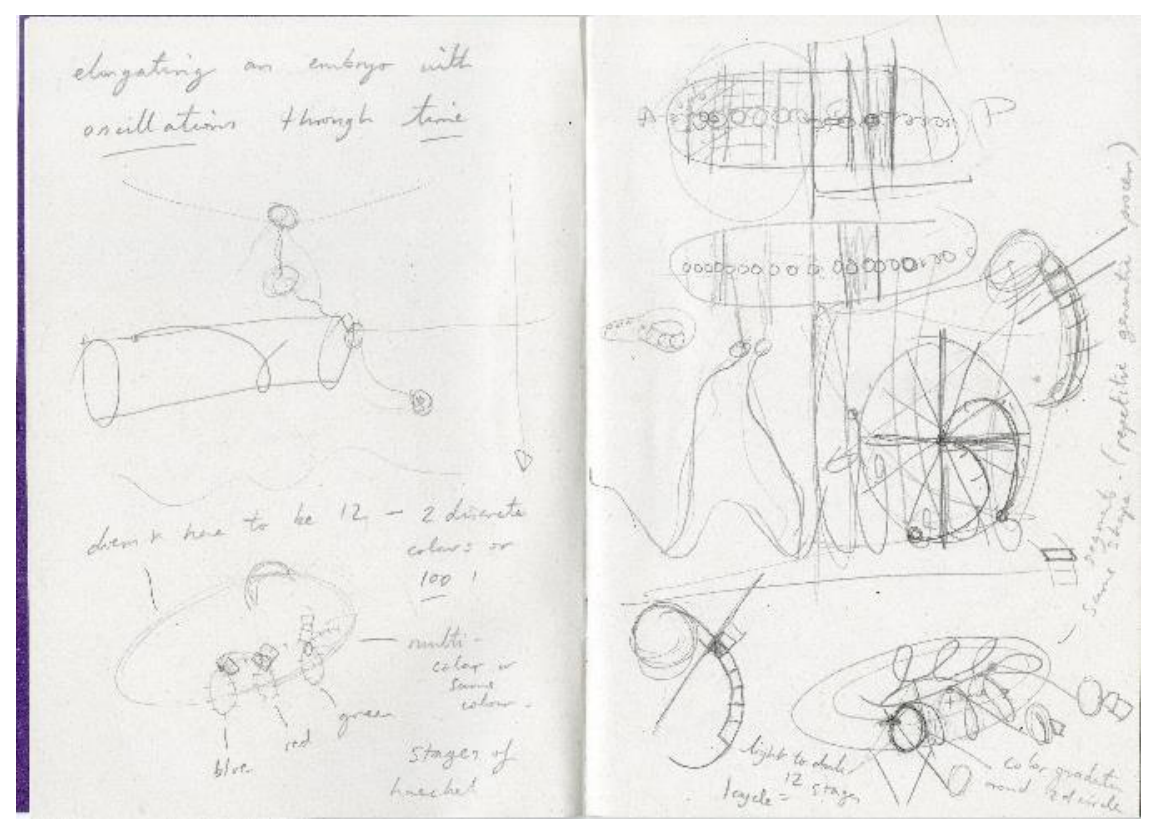

Fig. 4. Collaborative drawings: Gemma Anderson \& Berta Verd, "Somitogeneis/Oscillations." Pencil on paper, 2016. (@) Gemma Anderson)

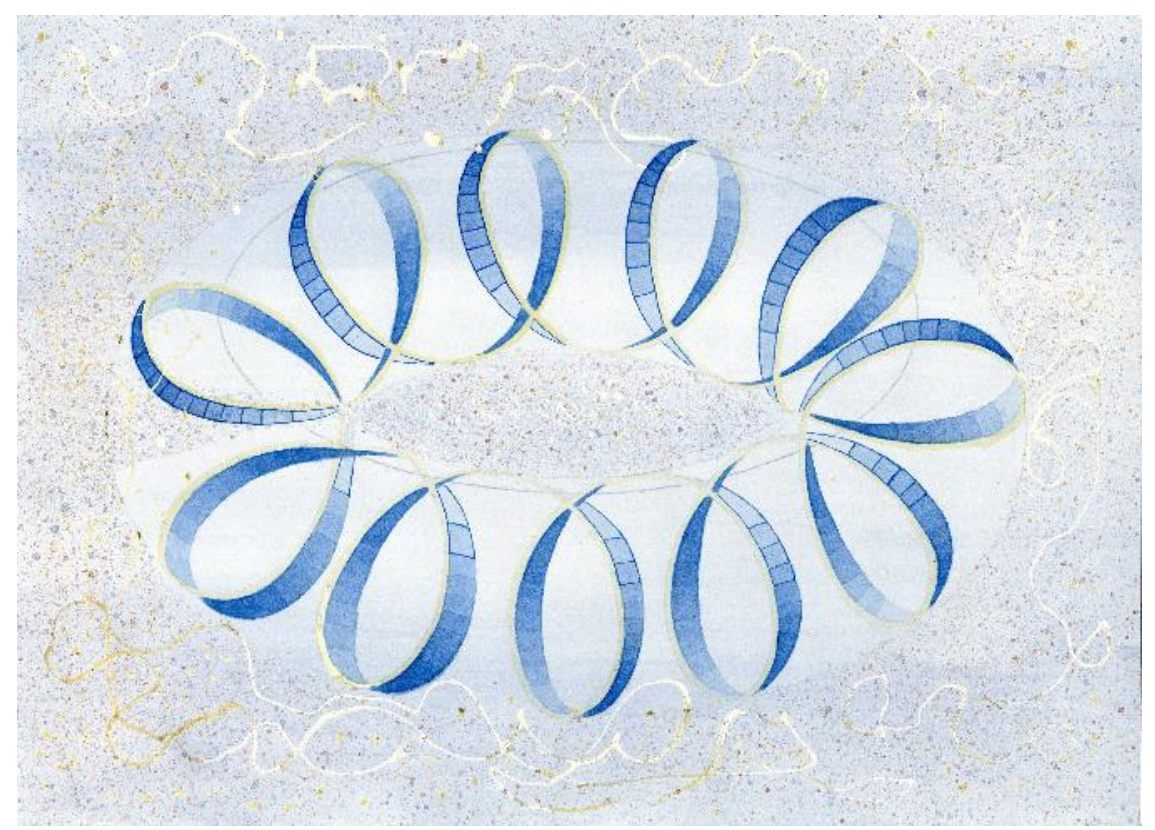

Fig. 5. Gemma Anderson, "Somitogeneis/Oscillations:Torus." Watercolour and pencil on paper, 2016. (C) Gemma Anderson)

The torus topology turned out to be extremely useful as a visual tool for thought. It successfully marries mathematical intuition and observation (landscape and oscillations), by releasing the landscape from the constraint of a strictly two-dimensional surface. It allows us to experiment with various parameters like the frequency and period of the oscillations, or the diameter and length of the torus, which creates intuitions for comparing the formation of somites in different organisms, from snakes to mice. In this sense, the image constitutes a powerful new visualisation tool to think about the evolution of vertebrate somitogenesis. 
'Somitogenesis/Oscillations:Torus' is the final product of Anderson's first visit to the KLI. Although satisfied with the advances provided by this image, we were still acutely aware of its limitations, as it prioritises gene expression patterns over an accurate representation of morphological transformations. In April 2017, Anderson returned to the KLI in the context of a residency provided by KulturKontakt Austria. In the meantime, Anderson had developed a more systematic approach to creating and depicting developmental noise. Her drawing system 'Noise/Form/Gradation' provides the formal components for creating image texture with relationships analogous to biological complexity [20]. Examples of this technique are shown in the images that follow. In addition, we wanted to experiment with even more complex topologies that would allow Anderson to introduce a more accurate representation of morphogenesis into her drawings. As a first step in this direction, we attempted to symbolize the complex, convoluted nature of organismic development by introducing knots into the torus (Fig. 6).

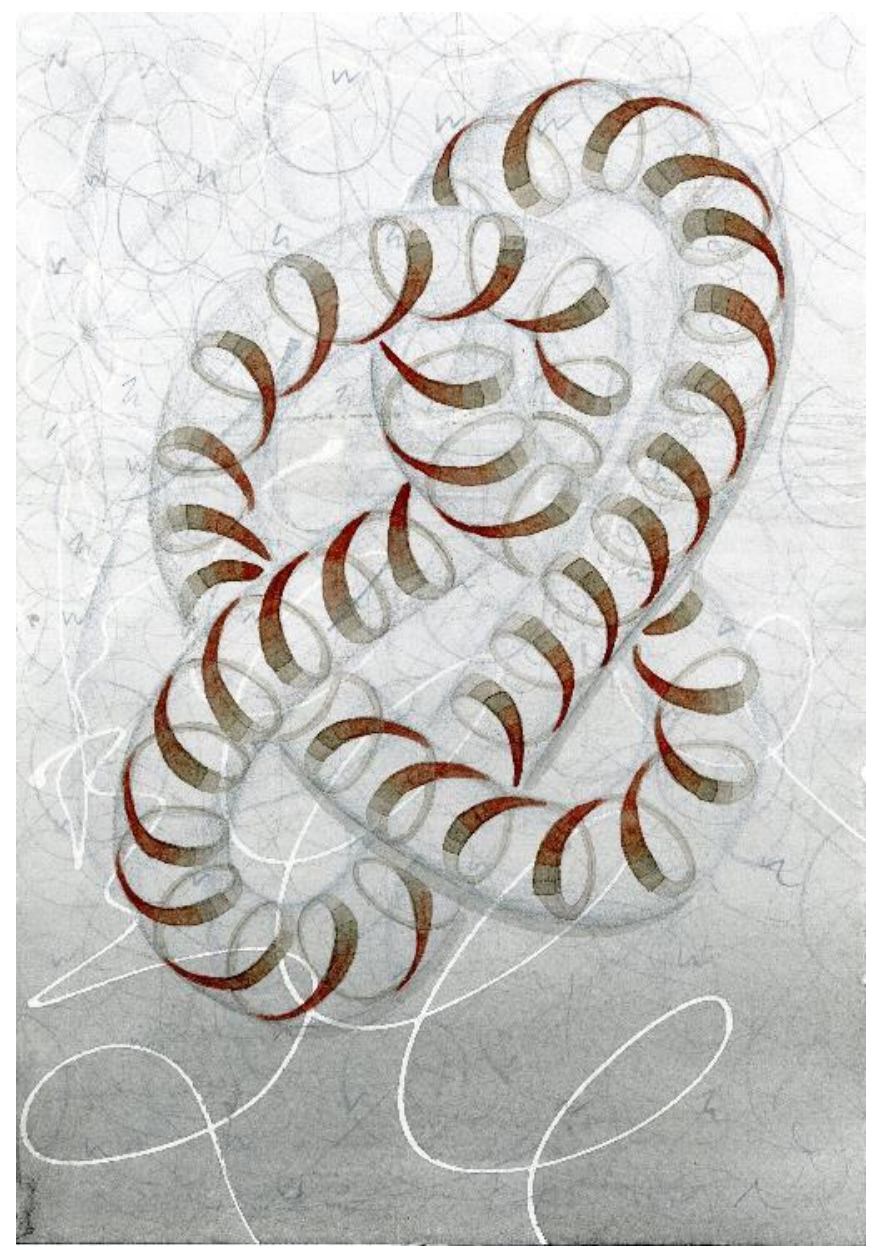

Fig. 6. Gemma Anderson, “Somitogeneis/Oscillations: Knot.” Watercolour on paper, 2017. (@ Gemma Anderson)

Knots deliver an increase in complexity, a more comprehensive exploration of the image space on the page, and an expansion of the surface area that can be occupied by the chreode. Different loops within the knotted structure could be thought of as representing different stages in a life cycle (embryo, larva, adult), each of which is subjected to variable levels and kinds of noise as 


\section{(C) 2019 ISAST}

indicated by the background. A complex knotted topology can also convey the sense of multiple oscillations happening at once, for instance, cell divisions and oscillatory waves of gene expression in the case of somitogenesis. Such combinations are extremely common in developmental biology. In all these ways, knotted loops provide an aesthetically pleasing compromise between complexity and simplicity. And yet, this representation still does not convey developmental trajectories in a truly realistic manner, as it does not incorporate branching or interacting paths. For this reason, Anderson decided to experiment with a network of spiralling tubes as a next step (Fig. 7).

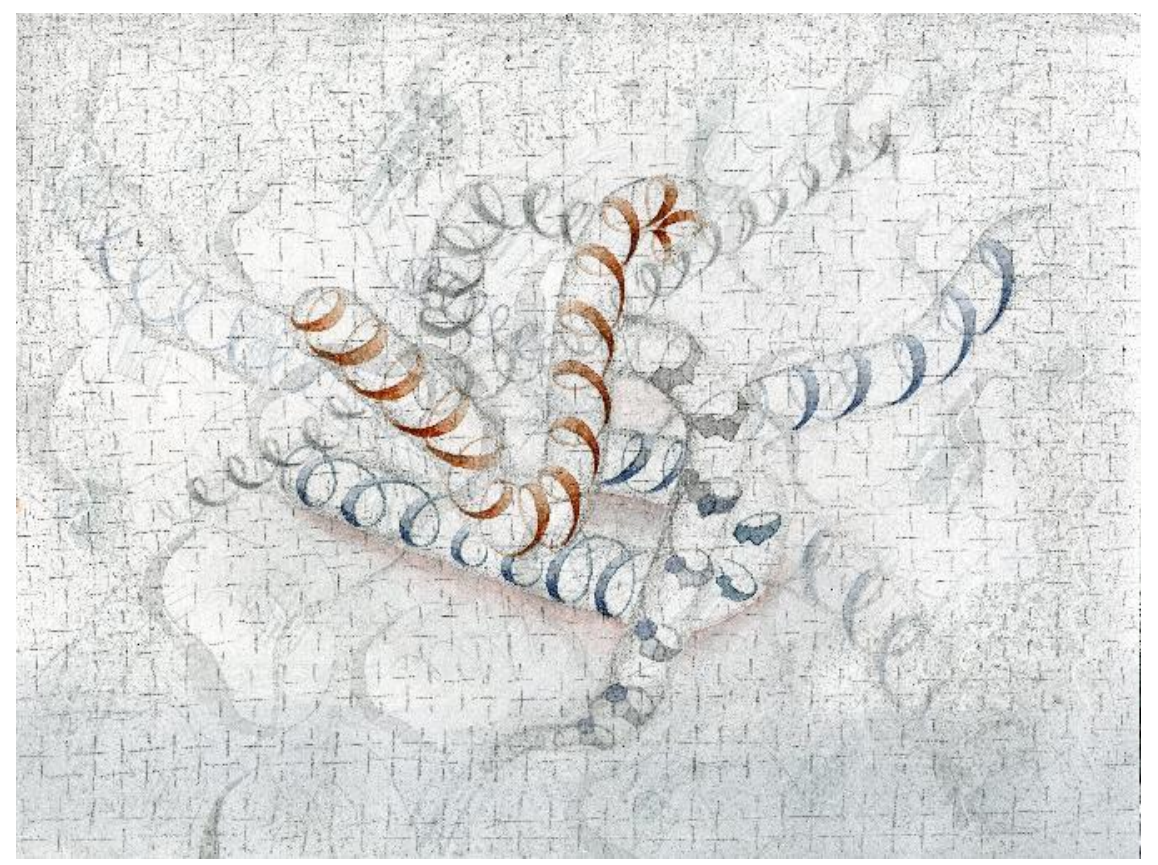

Fig. 7. Gemma Anderson, "Somitogeneis/Oscillations: Pathways." Watercolour on paper, 2017. (C) Gemma Anderson)

'Somitogeneis/Oscillations:Pathways' shows a tangled web of helical trajectories, coming in and out of focus. It highlights features of development complementary to those represented in the previous images. Figs. 5 and 6 convey the cyclic and periodic nature of biological processes. The trajectories shown in Fig. 7 may form loops as well, but only outside the focal plane of the image. Instead, different processes (marked through distinct colour gradation) connect and influence each other's direction and morphogenetic transformations (drawn as simplified Isomorphogenesis-like series in some of the grey-shaded tubes). This represents induction, an important and fundamental type of developmental event, in which one tissue signals to another to alter its fate. Waddington called inductive signalling "evocation" of a competent tissue [21]. An example of this type of interaction is the induction of lens formation by the optic cup during the development of the vertebrate eye [22].

A satisfactory synthesis of Figures 5, 6 and 7 requires a landscape topology combining cyclic aspects with different intertwined processes. Verd suggested a topological structure that includes both: the Klein Bottle [23]. Like its better-known cousin, the Möbius Strip, a Klein Bottle is a two-dimensional surface that has one side only. We can understand the Klein Bottle as having a main body, which narrows to form a "handle." The handle forms a cylindrical tube that intersects 


\section{(c) 2019 ISAST}

with the side of the main body looping back to connect the inside surface of the bottle to its outside at the bottle's base. This topology makes it possible for handle and main body to represent interactions of different processes within an intertwined cyclical trajectory (Fig. 8).

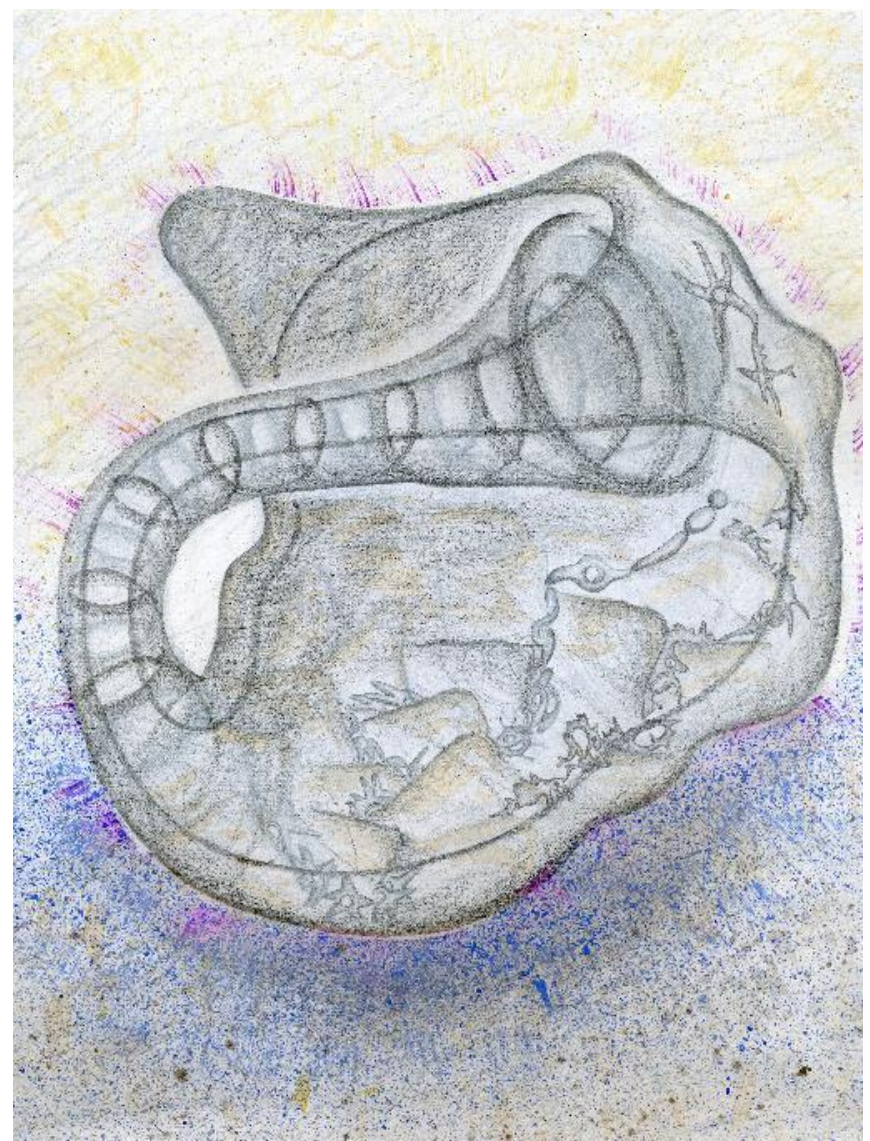

Fig. 8. Gemma Anderson, "Klein Bottle Landscape“. Watercolour and coloured pencil on paper, 2017. (@) Gemma Anderson)

The Klein Bottle provides a combination of torus and Waddington landscape, accommodating both oscillatory and branching dynamics. Handle and body of the bottle can be interpreted as different stages of development with fundamentally different levels of noise and canalization. The handle resembles one of the tubes in Figs 5-7. It contains a spiralling undifferentiated path, corresponding to early stages of embryogenesis. In contrast, the surface of the main body provides space for branching Isomorphogenesis transformations to unfold across an underlying Waddington landscape with valleys and peaks. This landscape is drawn on the "outside" surface of the body of the bottle. The looping topology of the Klein bottle makes it possible to represent many iterations of these processes, a representation of the life cycle of an organism.

There are vastly different levels of developmental noise on different sides of the bottle. The representation of canalization and noise is much more sophisticated in this image compared to Figs 5-7. Not only extrinsic noise levels vary, but also the nature of intrinsic canalizing constraints. The narrow cylinder of the handle prevents branching morphogenesis, but allows for variation in the period or amplitude of the oscillation. It is difficult to predict where exactly a specific cell will exit the handle. This represents the stochastic behaviour of undifferentiated 
stem cells [24]. On the main body of the bottle, branching morphogenesis does occur, but is tightly canalized by the topography of the landscape on its outer surface. It represents the more diversified, but also more canalized, late development of distinct differentiating cell populations.

In summary, the Klein bottle allows us to represent a complex knotted cyclical path with a representation of branching morphological transformations. From an artistic point of view, it enables Anderson to extend her Isomorphogenesis method onto a landscape in combination with a depiction of stochastic oscillatory dynamics in the handle of the bottle. Seen in this light, Fig. 8 provides a powerful synthesis of our explorations into a realistic, yet still intuitive, pictorial representation of developmental dynamics through our collaborative image-making process. In addition, it demonstrates the power of using complex topologies to represent high-dimensional developmental dynamics.

\section{Conclusions}

Our collaborative approach to extend Waddington's Landscape through drawing is an on-going iterative and creative process that lives in the space between art and science. The upshot of this process is not the illustration of a scientific concept, but to gain novel insights into the nature of life's processes. This does not happen through explicitly formulated conceptual analysis, as is usual within the framework of the scientific method, but rather through absorption of our images "by in-feeling" as Waddington fittingly puts it in the opening quote to our paper. It is a two-way exchange to which artist and scientist contribute equally. On the one hand, drawing is used as an epistemological tool and point of convergence that enables both artist and scientist to jointly develop their knowledge of the world. It provides images to think with. Complex landscape topologies, such as tori and Klein Bottles, enable Verd and Jaeger to develop new intuitions and concepts regarding the transformation of morphogenesis during evolution. This is achieved through new techniques for the visualization of dynamic processes, but also the contemplative, interactive, and iterative process of drawing itself. Drawing allows us to selectively highlight and explore salient features of a phenomenon. It is also a powerful constructive method for sharing insights across disciplines. On the other hand, our project reveals how the artist can make use of scientific concepts and processes - such as complex landscape topologies to represent oscillatory dynamics - to develop new approaches and methods for the visualization of dynamic form.

Modern neuroscience confirms the age-old intuition that creativity-the formation of new associations and concepts - requires playful abandoning of preconceived notions. To be creative we must let our minds wander [25]. Sadly, our rush for productivity, and the loss of space and time for contemplative practices, especially the decline of drawing in scientific practice, make this sort of creative work increasingly hard to achieve. This hampers the creative potential of modern scientific inquiry. Our collaborative image-making process is an effort to re-establish focused creative contemplation in scientific practice. Hand-drawing must not be allowed to disappear. We say this not because of its use in scientific illustration, but because of its potential as an epistemological tool to form insight and understanding, to share this understanding, and to enhance creativity among artists and scientists alike.

\section{References}




\section{(C) 2019 ISAST}

References are provided below. A more comprehensive supplementary bibliography with notes is available online upon print publication.

[1] C. H. Waddington, "New Visions of the World," Leonardo, 1, 69-77 (1968) p. 72.

[2] C. H. Waddington, Tools for Thought (New York, Paladin, 1977) p. xiii. B. Wittmann, "Knowledge in the making: drawing and writing as research techniques," Berlin, MPI for the History of Science, knowledge-in-the-making.mpiwgberlin.mpg.de/knowledgeInTheMaking/en/index.htm (2011) accessed on July $22^{\text {nd }} 2015$.

[3] C. H. Waddington, "New Visions of the World," Leonardo, 1, 69-77 (1968) p. 74.

[4] G. Anderson, "Endangered: a study of morphological drawing in zoological taxonomy," Leonardo 47, 232-40 (2014). G. Anderson, D. Buck, T. Coates \& A. Corti, "Drawing in mathematics: from inverse vision to the liberation of form," Leonardo 48, 439-48 (2015). G. Anderson, Drawing as a Way of Knowing in Art and Science. (Bristol, Intellect Press, 2017).

[5] J. Spiller, ed., H. Norden, transl., The Paul Klee Notebooks: The Nature of Nature (London: Lund Humphries, 1973).

[6] C. H. Waddington, An introduction to modern genetics (New York: Macmillan, 1939). C. H. Waddington, Organisers \& genes (Cambridge: Cambridge University Press, 1940). C. H. Waddington, The Strategy of the Genes (London, Allen \& Unwin, 1957).

[7] See also A. D. Goldberg, C. D. Allis \& E. Bernstein, "Epigenetics: a landscape takes shape", Cell 128, 635-8 (2007). D. Sareen \& C. N. Svendsen, "Stem cell biologists sure play a mean pinball", Nature Biotechnology 28, 333-5 (2010). D. Noble, "Conrad Hal Waddington and the origins of epigenetics", Journal of Experimental Biology 218, 816-8 (2015). In contrast to these efforts, we focus on representing complex developmental dynamics in a more accurate and realistic way.

[8] C. H. Waddington, "Canalization of development and the inheritance of acquired characters", Nature 150, 563-5 (1942).

[9] J. Baedke, "The epigenetic landscape in the course of time: Conrad Hal Waddington's methodological impact on the life sciences", Studies in History and Philosophy of Biological and Biomedical Sciences 44, 756-73 (2013). G. Fusco, R. Carrer \& E. Serrelli, “The landscape metaphor in development", in: A. Minelli \& T. Pradeu, eds., Towards a Theory of Development (Oxford, Oxford University Press, 2014). B. K. Hall, "Waddington's legacy in development and evolution", American Zoologist 32, 113-22 (1992). S. F. Gilbert, "Epigenetic landscaping: Waddington's use of cell fate bifurcation diagrams", Biology \& Philosophy 6, 135-54 (1991). S. F. Gilbert, "Diachronic biology meets evo-devo: C. H. Waddington's approach to evolutionary developmental biology", American Zoologist 40, 729-37 (2000). J. M. W. Slack, "Conrad Hal Waddington: the last Renaissance biologist?", Nature Reviews Genetics 3, 889-95 (2002). E. L. Peterson, "The excluded philosophy of evo-devo? Revisiting C. H. Waddington's failed attempt 
to embed Alfred North Whitehead's 'Organicism' in evolutionary biology", History and Philosophy of the Life Sciences 33, 301-20 (2011).

[10] R. Thom (1976). Structural stability and morphogenesis (Reading, MA, W. A. Benjamin Inc., 1976).

[11] M. B. Fagan, "Waddington redux: models and explanation in stem cell and systems biology", Biology \& Philosophy 27: 179-213 (2012).

[12] S. Huang. "The molecular and mathematical basis of Waddington's epigenetic landscape: a framework for post-Darwinian biology? BioEssays 34, 149-57 (2012). B. Verd, A. Crombach \& J. Jaeger, "Classification of transient behaviours in a time-dependent toggle switch model", $B M C$ Systems Biology 8: 43 (2014). J. Jaeger \& N. Monk, "Bioattractors: dynamical systems theory and the evolution of regulatory processes", Journal of Physiology 592, 2267-81 (2014).

[13] C. H. Waddington, "New Visions of the World”, Leonardo, 1: 69-77 (1968), p. 74.

[14] The abstract nature of the landscape is highlighted by Slack (2002) who calls it a "colourful metaphor" with, as Gilbert (1991) points out, "no grounding in physical reality." See [9] for references.

[15] See, for example, J. E. Ferrell, "Bistability, bifurcations, and Waddington's epigenetic landscape", Current Biology 22, R458-66 (2012).

[16] B. C. Goodwin, Temporal Organization in Cells (Academic Press, London, 1963). A. Goldbeter, Biochemical Oscillations and Cellular Rhythms (Cambridge, Cambridge University Press, 1996). A. Winfree, The Geometry of Biological Time, $2^{\text {nd }}$ ed., (Springer: New York, 2001). A. Aulehla \& O. Pourquié, "Oscillating signaling pathways during embryonic development", Current Opinion in Cell Biology, 20, 632-637 (2010). A. Isomura \& R. Kageyama (2014, "Ultradian oscillations and pulses: coordinating cellular responses and cell fate decisions", Development 141, 3627-36 (2014).

[17] A. C. Oates, L. G. Morelli \& S. Ares, "Patterning embryos with oscillations: structure, function and dynamics of the vertebrate segmentation clock", Development 139, 625-39 (2012).

[18] G. Anderson, Drawing as a Way of Knowing in Art and Science (Bristol, Intellect Press, 2017). For D'Arcy Thompson's theory of transformations, see chapter IX of D'Arcy Wentworth Thompson, On Growth and Form (abridged Canto Edition) (Cambridge: Cambridge University Press, 1992). Klee's colour gradations are described in P. Klee, Notebooks Volume 2: The Nature of Nature, J. Spiller, ed.; H. Norden, transl. (New York: Wittenborn, 1970). Latham's "FormSynth" system for the generation of form is described in W. Latham, "Mutator 1+2: Evolutionary art by William Latham", http://www.phoenixbrighton.org/archive/2013-2/williamlatham-mutator-1-2 (2014, accessed: September 2015).

[19] See [17]. 
[20] Noise/Form/Gradation is a systematic approach, developed by Anderson (see [18]), which is used to simulate artistic compositions that are analogous to developmental themes.

[21] For embryonic induction, see S. F. Gilbert, Developmental Biology, $11^{\text {th }}$ ed. (Sunderland, MA: Sinauer Associates, 2016). Waddington's notion of "evocation" is first described in C. H. Waddington, Organisers \& Genes (Cambridge: Cambridge University Press, 1940).

[22] A. Cvekl \& R. Ashery-Padan, "The cellular and molecular mechanisms of vertebrate lens development", Development 141, 4432-47 (2014).

[23] mathworld.wolfram.com/KleinBottle.html (accessed on Oct $4^{\text {th }}$ 2017).

[24] N. Moris, C. Pina \& A. Martinez Arias, “Transition states and cell fate decisions in epigenetic landscapes", Nature Reviews Genetics 17, 693-703 (2016).

[25] See, for example, B. W. Mooneyham \& J. W. Schooler, "The costs and benefits of mindwandering: a review", Canadian Journal of Experimental Psychology 67, 11-18 (2013). See also review in E. Kandel, The Age of Insight: The Quest to Understand the Unconscious in Art, Mind, and Brain, from Vienna 1900 to the Present (New York: Random House, 2012). 